\title{
Assessment of Stage 6 object permanence
}

\author{
ROBERT PASNAK, MAURA KURKJIAN, and ESTRELLA TRIANA \\ George Mason University, Fairfax, Virginia
}

\begin{abstract}
The sensorimotor intelligence of cats and dogs was tested via successive invisible displacement object-permanence tasks. Preferred foods were used as baits, and a deception technique was used to control for odor cues. Dogs tested in their homes solved the problems consistently, but cats were seldom successful. It appears that dogs have fully developed sensorimotor intelligence, at least as indicated by object permanence. There is as yet no good evidence that cats have advanced beyond the fifth stage.
\end{abstract}

Dore (1986) recently published two creative and interesting experiments intended to measure the sensorimotor intelligence, as defined by Piaget, of adult cats. He found that cats solved successive visible displacement object-permanence tasks, but failed tasks that he termed "single invisible displacements." He concluded that the cognitive development of cats had progressed beyond the fourth stage of sensorimotor intelligence to the fifth stage, but not to the sixth. This conclusion differs from that of Gruber, Girgus, and Banuazizi (1971), who reported that the fourth stage was the upper limit for cats. It also differs from that of Triana and Pasnak (1981), who reported that cats could solve both single and successive invisible displacement tasks, and hence had completed the sixth and last stage in the development of sensorimotor intelligence. Thus these three studies published on cat's sensorimotor intelligence led to three different conclusions.

The pioneering work of Gruber et al. (1971) clearly showed that cats have reached the fourth stage. However, Triana and Pasnak (1981) suggested that the soft toys used as targets in that study were not sufficiently motivating for Stage 6 tasks, and Gruber et al.'s study included no Stage 5 tasks. The experiments by Dore (1986) and Triana and Pasnak (1981) showed that sufficiently motivated adult cats can solve Stage 5 tasks. It appears, then, that the real issue is whether the limit of cats' sensorimotor intelligence is Stage 5 or Stage 6.

The distinction between Stages 5 and 6 is especially important for understanding the intelligence of species, inasmuch as Stage 6 demarcates the beginning of symbolic thought in human infants. The single invisible displacement task is contrived so as to require the subject to infer movements of an object that cannot be sensed, and so indicates an early form of such thought. The subject must watch as an object is entirely concealed in the experimenter's hand or in a container. The hand or container is next moved entirely under or behind a screen, then taken back out and shown to be empty (or the subject is allowed to discover it to be empty). No possibility exists except that the object has been dropped under or behind the

Reprint requests should be addressed to Robert Pasnak, Department of Psychology, George Mason University, Fairfax, VA 22030. screen (unless the subject is sophisticated enough to assume false compartments, sleight of hand, or other deceptions). The subject that searches under or behind the screen is said to have "an actual image of the itinerary followed by an object" that cannot be sensed (Piaget, 1937/1954, p. 82). When children solve this task, an internal representation of the object is posited as the basis for their inference that the object must have been dropped under the screen. Such internal representation, which becomes progressively elaborated until the path of a concealed object can be followed not only to one but through a series of hidings (displacements), is a key feature of Stage 6 .

In Stage 5, movements of an object cannot be deciphered unless the moving object is directly observed. Stage 5 infants are considered to lack the ability to represent mentally an unsensed object (one concealed in a container) as it is moved about, and hence to lack the ability to solve invisible displacements.

Dore's (1986) experiments showed that cats can easily solve successive visible displacements, which are usually considered to mark the culmination of Stage 5. His conclusion that cats cannot produce valid solutions of single invisible displacements appears to be unwarranted, however, because his invisible displacement task was impossible to solve except by artifact (practical search). Because of an apparatus problem, Dore could not move the container holding the target object (a metal-capped dowel) under any cover. Instead the container stopped in front of the cover and, unseen by the cat, a magnet drew the dowel under the cover. Subsequently the cat was allowed to see or discover that the container was empty. This defeats the logic of the invisible displacement task. If the subject has no knowledge of some way in which the dowel could move itself, the subject will assume that it cannot be under the cover, since the container holding it never got there. Cats or infants should be baffled by this problem, since mental representation of the dowel's path requires the conclusion that it must be in the container and cannot be under the cover. With no logical explanation for the dowel's disappearance, the subject might search everywhere, or in the vicinity of the container or near its path, because there is no better alternative; this appears to be what Dore's cats did. 
It may be noteworthy that the percentage of "correct" solutions jumped from $4 \%$ to $27 \%$ when the container passed behind the covers in one of Dore's procedures. Since the container could not be seen for a short period of time, it was possible that it had gone under the cover (it had not), so the subjects had more reason to picture that as the dowel's path. Again, a procedure whereby the container is known to have taken the object under the cover and come out empty is required to assess a cat's ability to solve single invisible displacements.

It may also be important that Dore's (1986) cats had varied experience with the dowel. All had three 30-trial sessions in which the dowel was simply an inanimate stick that never moved of its own accord. Some of the cats had no other experience with the dowel, whereas others had visible displacement trials in which the stick was seen to move, apparently of its own power (actually by magnetism), under one or more covers. The latter cats were much more successful in solving the subsequent invisible displacement tasks. Although Dore attributed the difference to these cats' experience that the stick had often been found under covers, it is also evident that they had had an opportunity to learn that the stick could move itself, making the "impossible" single invisible displacement task more understandable. If such learning did occur, it made it possible for the cats to infer that the stick moved itself from inside the container to under the cover. However, such an inference is much more complex than the inference required for human infants to solve invisible displacements. This further clouds the interpretation of Dore's tasks as indices of Stage 6 object permanence.

A third problem is that the visibly moving stick was the kind of toy cats like to capture, adding to their interest and incentive on the visible tasks. This enticement was not present on the invisible tasks, and its absence could account for differences in performance on the Stage 5 and Stage 6 tasks-a difference due to motivation, rather than cognition. These differences in incentive and opportunity for learning about self-moving targets, combined with the even more important apparatus problems, could explain the differences in results between Dore's (1986) visible and invisible displacement tasks, between the two experiments reported, and between the subject groups within experiments.

It would be premature to discount as yet the possibility that cats are able to understand invisible displacements.

On the other hand, Triana and Pasnak's (1981) experiments did not conclusively show that cats have Stage 6 object permanence, contrary to their assertions. Triana and Pasnak followed the standardized procedure of Uzgiris and Hunt (1975), and administered tasks in ascending order. The ascending trials are usually employed because without the warm-up effects and encouragement of previous successes, human infants too often fail at tasks that are below their true level of cognitive attainment. However, Dore (1986) pointed out quite rightly that invisible displacements can be solved by generalized search strategies if there are many preceding tasks.
In the present study we tested this possibility by utilizing Triana and Pasnak's (1981) procedure for the task that defines mastery of Stage 6-successive invisible displacement-without any preliminary trials. This procedure, described in detail below, employs a preferred food as the target object to increase motivation, and utilizes a deception to control for odor cues.

\section{METHOD}

\section{Subjects}

Five dogs and 3 cats-all mixed breeds-participated in trials conducted at an animal shelter. Three Labrador retrievers, 1 German shepherd, 1 Abyssinian cat, and 5 mixed-breed cats were used for trials conducted in the animals' homes. All animals were experimentally naive adults.

\section{Apparatus \\ Upright wooden right triangles, $30 \times 40 \mathrm{~cm}$, and $30 \times 30 \mathrm{~cm}$ cloth- covered platforms with 12-cm legs similar to those used by Dore (1986)} and Triana and Pasnak (1981) were employed as covers.

\section{Procedure}

Each animal was allowed to taste tuna in water, tuna in oil, cooked hamburger, and cooked chicken, and the preferred food was used as bait. (Cats frequently will not work for any but a preferred food, even if deprived for $24 \mathrm{~h}$ or more.)

After a brief (5-min) adaptation period, the preferred food was hidden under one of three cloth-draped covers arranged in a row in the frontal plane on the first two trials. On the next two trials, it was hidden behind one of three upright wooden triangles arranged similarly. These different covers were employed to reduce incidental learning effects. The following procedure was used: The food was displayed under the animal's nose and then entirely concealed in an experimenter's hand. While the animal was lightly restrained, the hand was placed entirely under (behind) one cover, came out into plain view again, went under (behind) the second cover, came out again, disappeared under (behind) the third cover, and then was displayed, empty, before the animal's face. The hand remained available for exploration for a few moments after the animal was released to search for the food and then was withdrawn to the experimenter's lap. The order in which the hand visited the covers was counterbalanced: left $(L)$, center $(C)$, right $(R)$; R, C, L; C, L, R; C, R, L. The experimenter always sat behind the center cover, and always dropped the food under (behind) the second cover visited. To be considered successful, the animal had to search the third cover before any other. In so doing, the animal had to move away from the experimenter and the cover where the food had actually been dropped and was presumably emitting odors. Such a solution requires the mental representation that the food has been moved in the path followed by the hand, and has been left under the last cover. The deception of dropping the food elsewhere is simply a control for odor cues. The animal would have to ignore them or be insensitive to them to solve the problem by searching the third cover first.

\section{RESULTS}

The 5 dogs and 3 cats tested in the animal shelter never solved the problems (see Table 1). It appeared (subjectively) that they were too excited to pay attention to the task. This may be a problem whenever such animals are brought from cages to this type of testing situation. The 4 dogs tested in their homes were always successful. Only one of the 6 cats tested in their homes solved each successive invisible displacement task. This cat's solutions were hesitant and were not repeated when the series of problems was readministered as an ad hoc check. A sec- 
Table 1

Solutions of Successive Invisible Displacements: Cover Selected First

\begin{tabular}{|c|c|c|c|c|c|c|c|c|c|}
\hline \multirow[b]{2}{*}{ Dogs } & \multicolumn{4}{|c|}{ Trials } & \multirow[b]{2}{*}{ Cats } & \multicolumn{4}{|c|}{ Trials } \\
\hline & 1 & 2 & 3 & 4 & & 1 & 2 & 3 & 4 \\
\hline \multicolumn{10}{|c|}{ Home Trials } \\
\hline 1 & 3 & 3 & 3 & 3 & 1 & $\mathbf{N}$ & $\mathbf{N}$ & 1 & $\mathbf{N}$ \\
\hline 2 & 3 & 3 & 3 & 3 & 2 & 2 & 3 & 3 & 1 \\
\hline 3 & 3 & 3 & 3 & 3 & 3 & 3 & 3 & 3 & 3 \\
\hline 4 & 3 & 3 & 3 & 3 & 4 & $\mathbf{N}$ & $\mathbf{N}$ & $\mathbf{N}$ & $\mathbf{N}$ \\
\hline & & & & & 5 & $\mathbf{N}$ & $\mathbf{N}$ & 1 & 2 \\
\hline & & & & & 6 & $\mathbf{N}$ & $\mathbf{N}$ & $\mathbf{N}$ & $\mathbf{N}$ \\
\hline \multicolumn{10}{|c|}{ Shelter Trials } \\
\hline 5 & $\mathbf{N}$ & $\mathbf{N}$ & $\mathbf{N}$ & $\mathbf{N}$ & 7 & $\mathbf{N}$ & $\mathbf{N}$ & $\mathbf{N}$ & $\mathbf{N}$ \\
\hline 6 & $\mathbf{N}$ & $\mathbf{N}$ & $\mathbf{N}$ & $\mathbf{N}$ & 8 & $\mathbf{N}$ & $\mathbf{N}$ & $\mathbf{N}$ & $\mathbf{N}$ \\
\hline 7 & $\mathbf{N}$ & 1 & $\mathbf{N}$ & 2 & 9 & $\mathbf{N}$ & $\mathbf{N}$ & $\mathbf{N}$ & $\mathbf{N}$ \\
\hline 8 & $\mathbf{N}$ & $\mathbf{N}$ & $\mathbf{N}$ & $\mathbf{N}$ & & & & & \\
\hline 9 & 1 & $\mathbf{N}$ & $\mathbf{N}$ & $\mathbf{N}$ & & & & & \\
\hline
\end{tabular}

Note-1, 2, 3, and $\mathrm{N}$ represent search of first, second, third, and no cover, respectively. Choice of the third cover was correct.

ond cat was successful on the second and third trials, but the others had no successes. We conclude that success is the exception, not the rule, for cats tested in this manner. The ascending order of test trials used by Triana and Pasnak (1981) at least had the effect of greatly regularizing their data; indeed, most or all of the solutions they reported may have been due to practical search. Their cats may have learned to go to the last cover visited by the experimenter's hand. Thus, there is as yet no good evidence that cats can solve successive invisible displacements, and hence no evidence that they have completely developed Stage 6 sensorimotor intelligence.

\section{DISCUSSION}

The difference in the performance of cats and dogs is surprising, and it is probably still too early to form a final conclusion about whether cats can solve successive invisible displacements. Not the least of the problems is that we do not know whether it is the individuals that perform best or those that give typical performances that define the limit for the species. Additionally, human analogue problems, whether using food as a bait or not, may be inadequate to test the sensorimotor intelligence of nonhuman species. The use of food with deception to control for odor cues is problematic. If the animal searches logically, as it approaches the "correct" cover, the odor that should confirm the correctness of the choice does not emerge, it will not find the food, and search should extinguish. On the other hand, use of a secondary reinforcer as a target can cause many failures because of insufficient motivation. Hence, the motivation of the animal during testing with the secondary reinforcer must be intense. Also, motivation during establishment of the secondary reinforcer, and the amount, frequency, schedule, and nature of the primary reinforcement used to establish it, should be manipulated so as to maximize the secondary reinforcer's incentive value. Another problem is that the successive invisible displacement task involves social knowledge that animals may lack: The only reason to infer that the target object has been dropped under the third cover instead of the other two is that after depositing the object, a reasonable experimenter would probably not keep making displacements. For these reasons, a different approach, using nonhuman analogue tasks such as Wise, Wise, and Zimmerman (1974) attempted, may be needed, even if the question of just what tasks will be convincing is not yet answered.

\section{REFERENCES}

Dore, F. (1986). Object permanence in adult cats (Felis catus). Journal of Comparative Psychology, 100, 340-347.

Gruber, H., Girgus, J., \& BanuAzIzI, A. (1971). The development of object permanence in the cat. Developmental Psychology, 4, 9-15.

Piaget, J. (1954). The construction of reality in the child (M. Cook, Trans.). New York: Basic Books. (Original work published 1937)

Triana, E., \& PasnaK, R. (1981). Object permanence in cats and dogs. Animal Learning \& Behavior, 9, 135-139.

UzGIRIs, I., \& HUNT, J. McV. (1975). Assessment in infancy: Ordinal scales of psychological development. Urbana: University of Illinois Press.

Wise, K., Wise, L., \& Zimmerman, R. (1974). Piagetian object permanence in the infant rhesus monkey. Developmental Psychology, 10, 429-437.

(Manuscript received for publication December 21, 1987.) 